\title{
Inteligencia emocional y deporte: situación actual del estado de la investigación
}

\author{
Emotional intelligence and sport: current state of research
}

\section{Inteligência emocional e esporte: situaçáo atual do estado da pesquisa}

\author{
Almudena Ros Martínez*, Francisco J. Moya-Faz* y Enrique J. Garcés de Los Fayos Ruiz** \\ *Universidad Católica San Antonio. Murcia. ${ }^{* *}$ Universidad de Murcia.
}

\begin{abstract}
Resumen: En este estudio se presenta una revisión del trabajo científico publicado, entre los ańos 2001 y 2012, sobre deporte e inteligencia emocional para conocer el estado de la cuestión de estas variables. La búsqueda se ha realizado a través de las bases de datos electrónicas, PsycINFO, SPORTDiscus, Academic Search Premier, Web of Science y Medline. Una vez seleccionados los artículos, se procedió al análisis de la siguiente información: número de artículos publicados, autores que participan en cada uno de los trabajos, países en los que más se trabaja esta temática, universidades, revistas científicas, metodología, variables relacionadas e instrumentos utilizados.

Palabras clave: inteligencia emocional, deporte, análisis bibliométrico.

Abstract: This study presents a review of published scientific work, from 2001 until 2012, about sport and emotional intelligence in sports to know the state of affairs of these variables. The search was performed through electronic databases, PsycINFO, SPORTDiscus, Academic Search Premier,
\end{abstract}

Web of Science and Medline. After selecting the items, we analyzed the most relevant information: number of published articles, authors involved in each work, in which more countries are working this issue, universities, scientific journals, methods, variables and instruments used.

Keywords: emotional intelligence, sport, bibliometric analysis.

Resumo: Esse estudo apresenta uma revisão do trabalho cientifico publicado entre os anos 2001 e 2012 sobre esporte e inteligência emocional, com o objetivo de conhecer o estado da questão das variáveis. A busca bibliográfica foi realizada através das bases de dados eletrônicas PsycINFO, SPORTDiscus, Academic Search Premier, Web of Science y Medline. Uma vez selecionados os artigos, foram realizadas as analises das seguintes informaçóes: número de artigos publicados, autores que participaram em cada um dos estudos, países que mias pesquisam sobre o tema, universidade, revistas científicas, metodologia, variáveis relacionadas e instrumentos utilizados. Palavras chave: Inteligência emocional, esporte, analise bibliográfico.

\section{Introducción}

La Psicología del Deporte y la Actividad Física es la rama de la psicología que estudia los procesos psíquicos y la conducta del hombre durante la actividad deportiva. Esta ciencia aplicada busca conocer y optimizar las condiciones internas del deportista para lograr la expresión del potencial físico, técnico y táctico adquirido en el proceso de preparación Se estudiarán los diferentes procesos psicológicos que están influyendo en el comportamiento de los deportistas tales como la motivación, los procesos emocionales y su influencia en el rendimiento de los deportistas, así como diferentes aspectos relacionados con la intervención de la psicología en el deporte de rendimiento, de iniciación y de salud.

De esta forma, el psicólogo del deporte ayuda al competidor a controlar el estrés, que produce una desorganización emocional y conductual que puede afectar su rendimiento.

Los enormes avances que se han producido en los últimos ańos en el estudio científico de la actividad física y el deporte se deben, principalmente, al interés y al esfuerzo de numerosos investigadores vinculados a las distintas disciplinas que configuran este apasionante campo. De hecho, en todas y cada una de estas disciplinas ha aumentado signifi-

Dirección para correspondencia

(abelen@ucam.edu). Universidad Católica San Antonio. Murcia. España. cativamente el número de trabajos teóricos y prácticos relacionados con el mundo del deporte, una feliz circunstancia que ha colocado a la Psicología del Deporte y a las Ciencias de la Actividad Física y del Deporte en un lugar de merecido reconocimiento. Dosil (2004), ya en el primer capítulo de su Psicología de la actividad Física y del Deporte, se refiere a este hecho subrayando que la reflexión y la investigación son absolutamente indispensables para entender la realidad de la que se ocupa una ciencia y, por ende, añadimos nosotros, para comprender y delimitar su ámbito de estudio y actuación. A grosso modo, especifica el autor que se pueden distinguir tres grandes etapas en la historia científica de la actividad física y el deporte:

1a) La actividad física y el deporte apenas si tienen interés científico, aunque sí interés social.

2a) La actividad física y el deporte comienzan a tener interés en los ámbitos que le son propios: Institutos $\mathrm{Na}$ cionales de Educación Física (INEF), Centros de Alto Rendimiento, Facultades de Ciencias de la Actividad Física y del Deporte, etc.

3a) La actividad física y el deporte ocupan un espacio científico más allá de los ámbitos que le son propios: facultades de sociología, psicología, medicina, ciencias de la información, etc. 
Tradicionalmente, los programas de preparación del deportista para el alto rendimiento han concedido mucha más importancia al progreso técnico y a la mejora de la forma física que al desarrollo de los factores psicológicos. Sin embargo, las múltiples exigencias de la competición, el elevado nivel de compromiso personal y la necesidad de rendir siempre al máximo han hecho que la preparación psicológica del deportista se convierta en algo tan necesario como puedan serlo la preparación física o la preparación técnica.

De hecho, como las diferencias en destreza y preparación física entre los atletas son cada vez menores, tendiendo a igualarse en la elite, los especialistas han llegado a la conclusión de que es la preparación psicológica la que permite al practicante "jugar con ventaja” y obtener el máximo rendimiento deportivo. Prueba de ello es que, actualmente, existe una conciencia creciente de que los factores psicológicos juegan un papel primordial en la ejecución motora de carácter deportivo.

No ha de sorprender, por tanto, que los profesionales del deporte se muestren cada vez más interesados en incorporar a sus programas de entrenamiento técnicas psicológicas que les ayuden a controlar el estrés de la competición, a mejorar la concentración, a aumentar la confianza en sí mismos y a incrementar el nivel de comunicación y la fluidez de las relaciones interpersonales dentro de los equipos deportivos.

Como señala Silva (1984), la clarificación de valores en los deportistas; el establecimiento de objetivos; la planificación del tiempo; el análisis de las atribuciones de éxito o fracaso; la evaluación del estrés, las fuentes de distracción y los momentos de máximo rendimiento; la mejora de la concentración; el aprendizaje de técnicas de relajación y de otras estrategias de afrontamiento en situaciones de competición son conocimientos indispensables para la preparación de entrenadores, jueces y deportistas.

No olvidemos, como indican Smith y Smoll (1991), que cualquier situación deportiva, ya sea de entrenamiento o de competición, puede servir como laboratorio natural para estudiar los procesos psicológicos básicos del deportista.

La Psicología del Deporte es, fundamentalmente, una “especialidad de la psicología cientifica que investiga y aplica los principios del comportamiento humano a las materias concernientes a la práctica del ejercicio físico y del deporte" (Lorenzo, 1997 , p. 35), una especialidad que no se limita al estudio del rendimiento, las destrezas psicomotoras o la personalidad del practicante, sino que se interesa por todos los aspectos psicológicos presentes en la práctica deportiva (inteligencia, voluntad, pensamientos, sensaciones, emociones, etc.) y por todas las interacciones que en ella tienen lugar.

Como señala Riera (1985), el psicólogo deportivo ha de tener la mentalidad del investigador que procura resolver los enigmas que observa, y aunque "en muchas ocasiones no podrá disponer del material, del tiempo, ni de las facilidades de acceso necesarias para estudiar cómo sería deseable las interacciones deportivas, ha de procurar mantener, por encima de todo, su criterio lo más cercano posible a la objetividad" (p. 72).

Los estudios sobre inteligencia emocional tienen una gran trayectoria desde 1995 pero no será hasta 2001 cuando aparezca literatura de esta variable aplicada al campo del deporte. Incluso no siendo muy productiva ya que se está empezando a investigar sobre la misma. Por lo que nos encontramos ante un campo de investigación poco explorado con las consecuentes puertas que se abren ante el investigador que quiere aplicar esta variable tan interesante al deporte.

En el alto rendimiento deportivo, las exigencias y demandas de la competencia son cada vez mayores. La psicología deportiva se encarga de analizar, estudiar y observar las conductas, reacciones y respuestas emocionales del individuo o equipo, al momento de la competencia. Para ello debe "controlar" positivamente sus emociones o re-direccionarlas de manera que no influyan negativamente en el gesto deportivo.

¿Y porque la inteligencia emocional? porque en el deporte de alto rendimiento hay que tomar decisiones extremadamente rápidas y adecuadas. Goleman (1995) entiende la emoción como "un sentimiento que afecta a los propios pensamientos, estados psicológicos, estados biológicos y voluntad de acción”. Las 6 habilidades de la inteligencia emocional son: valorar, autoconciencia, autocontrol, motivación, empatía y habilidad social.

La inteligencia emocional se puede aprender y así controlar emociones tan significativas en el mundo del deporte como la ansiedad, las presiones, los miedos (estrés) y la agresividad. Que muchas veces son perturbadoras y merman el rendimiento. La ansiedad es considerada una emoción típica del fenómeno del estrés según Spielberger y Guerrero (1975) quien define el estrés como "un complejo proceso psico-biológico que consiste de tres grandes elementos: estresores, percepciones, o evaluaciones de peligro (amenaza) y reacciones emocionales.

Teniendo en cuenta la poca productividad existente con las variables inteligencia emocional y deporte, consideramos necesario este estudio que se muestra como un procedimiento válido para conocer el estado de la cuestión de la inteligencia emocional en el contexto deportivo.

\section{Inteligencia emocional (I.E) y Deporte}

El mundo deportivo brinda muchas satisfacciones, a cambio de muchos sacrificios. El deporte, representa una manera de relajación, una forma de enfrentarse a retos, una oportunidad de colaboración en equipo o una competición con uno mismo. Cuando el marco es el del deporte de competición o profesional todos los comentarios anteriores se intensifican y además hay que sumarle los ańadidos de la profesionalidad, la excelencia, la presión y la proyección a la que cada uno aspire.

En el alto rendimiento deportivo, las exigencias y demandas de la competencia son cada vez mayores. La psicología deportiva se encarga de analizar, estudiar y observar las con- 
ductas, reacciones y respuestas emocionales del individuo o equipo al momento de la competencia. Para ello debe "controlar" positivamente sus emociones o re-direccionarlas de manera que no influyan negativamente en el gesto deportivo y favorezcan un proceso de toma de decisiones que ha de ser extremadamente rápido y eficaz. Daniel Goleman (1995) entiende la emoción como "un sentimiento que afecta a los propios pensamientos, estados psicológicos, estados biológicos y voluntad de acción”. Las 6 habilidades que componen la IE son (Goleman, 1995):

- Conocimiento de las propias emociones.

- Capacidad para motivarse a sí mismo.

- Capacidad para controlar las emociones.

- Capacidad para reconocer las emociones ajenas.

- Control de las relaciones interpersonales.

Por todo esto, la Inteligencia Emocional cobra mucho interés para este colectivo. Aquí los elementos tanto intrapersonales como los interpersonales tienen una aplicación directa. El conocimiento de uno mismo, la autorregulación de emociones, la automotivación, las habilidades sociales y la empatía son herramientas que todo deportista maneja en mayor o menor medida, bien sea consciente o inconscientemente.

La Inteligencia Emocional y el Deporte están íntimamente relacionados, hasta tal punto que muchas de las técnicas de relajación, concentración, visualización son compartidas y cada vez más los clubes, las federaciones e incluso los entrenadores están contratando más y más profesionales para que implanten estas técnicas y mejorar así el rendimiento de los deportistas.

Muchas de las herramientas que comparten la IE y el Deporte tienen la característica principal de ser tremendamente prácticas, sobre todo de aplicación en la vida diaria.

La importancia de que un atleta pueda ejercer control sobre sus emociones, con relación a la práctica deportiva se evidencia en diversos estudios (Biddle y Hill,1992;Gluch,199 3;Hanin,1993;Hanin,1995;Liukkonen,1995;Marciano,1989; Markowska et al.,1991;Ranglin,1995;Thomas,1994). Hannin y Sirja (1995) encontraron que las emociones en relación con el éxito deportivo presentas zonas óptimas individuales, pero no así a nivel grupal, claro está, cada atleta reacciona de una forma diferente ante el estrés requiriendo de cierto grado de emociones negativas o positivas que le den un punto óptimo de desempeño en situación de juego.

\section{Método}

La búsqueda de los artículos se realizo en las bases de datos: PsyINFO, SPORTDiscus, Academic Search Premier, Web of Science y Medline. Para sistematizar los datos obtenidos se diseñó una matriz que constituyó la base de datos general y que permitió identificar los siguientes elementos:
Autor (es) / País / Universidades / Año de publicación / Título / Metodología (teórico, empírico, instrumentos de evaluación...) / Revista científica / Instrumentos de evaluación / Variables psicológicas relacionadas en el estudio / Modalidades deportivas.

\section{Resultados}

El número de publicaciones encontradas sobre inteligencia emocional y deporte es de sesenta, comprendidos entre 2001 y 2012. En la tabla 1 se presentan las publicaciones por ańos entre los que se extiende el estudio. Como se puede ver en ella, la mayor productividad se produce a partir del 2009 siendo el año más productivo 2011.

Tabla 1. Publicación de trabajos por años (2001-2012)

\begin{tabular}{|ccc|}
\hline Año & No de publicaciones & $\%$ \\
\hline 2001 & 1 & 1.66 \\
\hline 2002 & 1 & 1.66 \\
\hline 2003 & 1 & 1.66 \\
\hline 2004 & 2 & 3.33 \\
\hline 2005 & 1 & 1.66 \\
\hline 2006 & 1 & 1.66 \\
\hline 2007 & 5 & 8.3 \\
\hline 2008 & 3 & 5 \\
\hline 2009 & 10 & 16.66 \\
\hline 2010 & 9 & 15 \\
\hline 2011 & 23 & 38.33 \\
\hline 2012 & 3 & 5 \\
\hline TOTAL & 60 & 100 \\
\hline
\end{tabular}

El primer trabajo referenciado por estas bases de datos que versa sobre inteligencia emocional y deporte data de 2001. Los ańos en los que se presenta mayor productividad son: 2009, 2010 y 2011, con diez, nueve y veintitrés publicaciones respectivamente, siendo el año 2011 el más productivo. En los últimos 5 ańos se han producido 48 trabajos de la producción total de sesenta. Los años con menos publicaciones son 2001, 2002, 2003, 2005 y 2006 con un solo trabajo publicado.

Se observa que desde el 2001 al 2008 la producción es casi nula siendo a partir del 2009 cuando hay un aumento considerable con respecto a los años anteriores. En definitiva, el $100 \%$ de los trabajos se ha publicado en la última década desde 2001 hasta 2012 siendo anterior a esta fecha inexistente este tipo de publicaciones en esta temática.

En relación con la productividad por paises (Tabla 2), podemos observar que el análisis de los 17 países incluidos refleja que casi la mitad de los trabajos corresponden a EEUU, lugar donde se efectuó el 20\% y al Reino Unido, donde se efectuó 
el $18.3 \%$ de toda la productividad. El resto, corresponde en su mayoría a estudios pertenecientes a 3 países (Australia, España y Sudáfrica) con seis y cuatro publicaciones respectivamente. Los restantes (12 en total) sólo han producido entre uno y tres trabajos. Destacamos España que ocupa el cuarto puesto en la lista con cuatro publicaciones junto con Sudáfrica.

Tabla 2. Productividad por países

\begin{tabular}{lcc} 
País & No publicaciones & $\%$ \\
\hline EEUU & 12 & 20 \\
\hline Reino Unido & 11 & 18.3 \\
\hline Australia & 6 & 10 \\
\hline Sudáfrica & 4 & 6.66 \\
\hline Espańa & 4 & 6.66 \\
\hline Canadá & 3 & 5 \\
\hline Turkia. & 3 & 5 \\
\hline Francia & 3 & 5 \\
\hline Iran & 2 & 3.33 \\
\hline Taiwan & 2 & 3.33 \\
\hline Slovenia & 2 & 3.33 \\
\hline China & 2 & 3.33 \\
\hline Grecia & 2 & 3.33 \\
\hline Finlandia & 1 & 1.6 \\
\hline Alemania & 1 & 1.6 \\
\hline Indianapolis & 1 & 1.6 \\
\hline Croacia & 1 & 1.6 \\
\hline
\end{tabular}

A continuación se analizó la productividad por autores. En los sesenta trabajos recogidos intervienen un total de 109 autores lo que supone una media de 1.85 autores por trabajo (Tabla 3).

Tabla 3. Relación autores y productividad

\begin{tabular}{lcc} 
Autor & No publicaciones & \% de publicaciones \\
\hline Lane, Andrew M & 8 & 5.59 \\
\hline Devonport, T. J & 6 & 4.19 \\
\hline Laborde, Sylvain & 3 & 2.09 \\
\hline Crombie, David & 3 & 2.09 \\
\hline León, Jaime & 3 & 2.09 \\
\hline Núnez, Juan L & 3 & 2.09 \\
\hline Thelwell, Richard & 3 & 2.09 \\
\hline Meyer, Barbara B & 3 & 2.09 \\
\hline You, Min & 2 & 1.39 \\
\hline Dosseville, Fabrice & 2 & 1.39 \\
\hline González, Víctor & 2 & 1.39 \\
\hline Wilson, Mathew & 2 & 1.39 \\
\hline Davies, KA & 2 & 1.39 \\
\hline Noakes, Timothy & 2 & 1.39
\end{tabular}

\begin{tabular}{lcc} 
Lombard, Carl & 2 & 1.39 \\
\hline Costarelli, V & 2 & 1.39 \\
\hline Zizzi, Sam & 2 & 1.39 \\
\hline Stough, C. & 2 & 1.39 \\
\hline Otros autores* & 91 & 63.48 \\
*Autores con un artículo. & &
\end{tabular}

*Autores con un artículo.

Siguiendo con los autores, en la tabla 4 presentamos la cantidad de autores según su número de firmas para el total de la muestra. No solo presentamos esto, sino que además distribuimos los autores en cuatro categorías según su productividad. Para realizar esta distribución seguimos la clasificación propuesta por Crane (1969; Quiles et al., 2000) quien establece lo siguiente: grandes productores (10 ó más trabajos), productores moderados (5-9 trabajos), aspirantes (2-4 trabajos) y transeúntes (1 trabajo).

Tabla 4. Número de autores según su productividad

\begin{tabular}{ccccc}
$\begin{array}{c}\text { No } \\
\text { Autores }\end{array}$ & $\begin{array}{c}\text { No } \\
\text { Articulos }\end{array}$ & $\begin{array}{c}\text { \% } \\
\text { Autores }\end{array}$ & Categoría & \% según categoría \\
91 & 1 & 83.49 & Transeúntes & 83.49 \\
10 & 2 & 9.17 & Aspirantes & \\
6 & 3 & 5.5 & Aspirantes & 14.67 \\
1 & 6 & 0.92 & Moderados & 1.84 \\
1 & 8 & 0.92 & & \\
\hline
\end{tabular}

Como puede observarse existen dos autores con mayor número de artículos: A.M. Lane, y T.J. Devonport con ocho y seis artículos respectivamente. Como productores moderados aparecen 2 autores. Como productores aspirantes nos encontramos con 16 autores que corresponde con $14.67 \%$ de los autores. Finalmente parece significativo que un $83.49 \%$, tengan un solo artículo, se trata de autores transeúntes.

Finalmente analizamos el nivel de colaboración en los artículos, es decir, el número de firmas por trabajo. Dentro de la productividad científica es interesante saber el número de autores que trabajan en colaboración (Tabla 5). El número de trabajos firmados por un solo autor es de 16 (27,12\%), el $28.81 \%$ lo firman dos autores, el $25.42 \%$ tres y cuatro el $10.17 \%$ y con cinco el $5.1 \%$ y siete o nueve el $3.4 \%$.

Tabla 5. Número de firmas por trabajo

\begin{tabular}{|ccc|} 
No de firmas por trabajo & No de trabajos & $\%$ \\
\hline 1 & 16 & 27.12 \\
\hline 2 & 17 & 28.81 \\
\hline 3 & 15 & 25.42 \\
\hline 4 & 6 & 10.17 \\
\hline 5 & 3 & 5.1 \\
\hline 7 & 1 & 1.7 \\
\hline 9 & 1 & 1.7 \\
\hline
\end{tabular}


Nos encontramos con una tendencia significativa a la colaboración como lo prueba el hecho de que el 54,23\% de los trabajos publicados hayan sido realizados por colaboración entre 2 ó 3 autores.

Para finalizar esta primera parte del análisis bibliográfico de la inteligencia emocional analizaremos la productividad por universidades (véase la Tabla 6). La información que se muestra en este apartado hace referencia a las instituciones donde los autores realizaron el trabajo. University of Wolverhampton de Reino Unido es la Universidad que mas artículos ha producido, 7. Les sigue con 2, Sports Science Institute of South Africa, University of Caen, Universidad de Las Palmas de Gran Canaria, Michigan State University y University of Wisconsin-Milwaukee con un total de 10 artículos. Con solo un artículo hay 21 Universidades.

Tabla 6. Número de trabajos por universidades

\begin{tabular}{lcc} 
Universidades & $\begin{array}{c}\text { No } \\
\text { trabajos }\end{array}$ & $\begin{array}{c}\% \\
\text { trabajos }\end{array}$ \\
\hline University of Wolverhampton & 7 & 18.42 \\
\hline Michigan StateUniversity & 2 & 5.2 \\
Universidad de Las Palmas de Gran Canaria & 2 & 5.2 \\
\hline Sports Science Institute of South Africa & 2 & 5.2 \\
\hline University of Caen & 2 & 5.2 \\
\hline University of Wisconsin-Milwaukee. & 2 & 5.2 \\
\hline Otras Universidades* & 21 & 55.26
\end{tabular}

*Universidades con un solo trabajo.

A continuación, en la tabla 7, se incluyen las revistas según el número de documentos que han publicado y su correspondiente porcentaje. Se trata de 16 revistas que contienen un único artículo, frente a 13 que tienen más de uno. La revista más productiva fue, International Journal of Sports Science \& Coaching con un total de 10 artículos.

Tabla 7. Número de artículos por revistas

\begin{tabular}{|c|c|c|}
\hline Revistas científicas & $\begin{array}{l}\text { No de } \\
\text { artículos }\end{array}$ & $\begin{array}{l}\% \\
\text { artículos }\end{array}$ \\
\hline $\begin{array}{l}\text { International Journal of Sports Science \& } \\
\text { Coaching }\end{array}$ & 10 & 18.1 \\
\hline $\begin{array}{l}\text { Social Behavior \& Personality: An Inter- } \\
\text { national Journal }\end{array}$ & 4 & 7.3 \\
\hline Journal of sport \& Exercise Psychology & 3 & 5.4 \\
\hline E-Journal of Applied Psychology & 3 & 5.4 \\
\hline Journal of Science \& Medicine in Sport & 3 & 5.4 \\
\hline $\begin{array}{l}\text { International Journal of Physical Educa- } \\
\text { tion }\end{array}$ & 2 & 3.6 \\
\hline Revista de Psicología del Deporte & 2 & 3.6 \\
\hline $\begin{array}{l}\text { Journal of Hospitality, Leisure, Sport \& } \\
\text { Tourism Education }\end{array}$ & 2 & 3.6 \\
\hline Journal of Teaching in Physical Education & 2 & 3.6 \\
\hline Perceptual and Motor Skills & 2 & 3.6 \\
\hline Personality and Individual Differences & 2 & 3.6 \\
\hline British journal of sports medicine & 2 & 3.6 \\
\hline
\end{tabular}

Journal of exercise science y fitness

Otras Revistas*

2

*Revistas con un artículo.

Referido al análisis según la metodología, recogemos todos los artículos publicados en revistas con un total de sesenta y los hemos dividido de la siguiente forma: trabajos teóricos, empíricos y estudio de casos e instrumentos de evaluación. En la tabla 8 presentamos tal distribución. Como puede apreciarse en dicha tabla, el $57.63 \%$ son empíricos repartiéndose el resto de manera bastante proporcionada: teóricos e instrumentos de evaluación con $42.37 \%$ y $44.07 \%$ respectivamente.

Tabla 8. Número de artículos según modalidad

\begin{tabular}{lcc} 
Metodología & No de artículos & $\%$ \\
\hline Empíricos & 34 & 57.63 \\
\hline Teóricos & 25 & 42.37 \\
\hline Instrumentos de evaluación & 26 & 44.07
\end{tabular}

En el total de artículos analizados se han estudiado 27 variables diferentes. En la tabla 9 se aprecia la distribución de los tipos de variables objeto de estudio. La variable que aparece en más ocasiones 9 es liderazgo. Le siguen rendimiento deportivo con 6 y estrategias de autorregulación, ansiedad y desarrollo y la mejora de su inteligencia emocional con 4 .

Tabla 9. Número de trabajos según la variable relacionada

\begin{tabular}{lcc} 
Variables & No & $\%$ \\
\hline Liderazgo & 9 & 14.75 \\
\hline Rendimiento deportivo & 6 & 9.84 \\
\hline $\begin{array}{l}\text { Desarrollo y la mejora de su inteligencia } \\
\text { emocional }\end{array}$ & 4 & 6.55 \\
\hline Estrategias de autorregulación & 4 & 6.55 \\
\hline Ansiedad & 4 & 6.55 \\
\hline Habilidades de afrontamiento & 3 & 4.92 \\
\hline Auto-conciencia & 3 & 4.92 \\
\hline Bienestar psicológico & 3 & 4.92 \\
\hline Inteligencia emocional percibida & 3 & 4.92 \\
\hline Las actitudes, los componentes de la ima- & 2 & 3.28 \\
gen corporal & 2 & 3.28 \\
\hline Orientación a la tarea & 2 & 3.28 \\
\hline Motivación percibida & 2 & 3.28 \\
\hline Perfiles de los activos de desarrollo & 1 & 1.64 \\
\hline Empatía & 1 & 1.64 \\
\hline Contagio emocional & 1 & 1.64 \\
\hline Actitudes sobre el ejercicio y el deporte & 1 & 1.64 \\
\hline Inteligencia emocional rasgo & 1 & 1.64 \\
\hline Estados emocionales negativos & 1 & 1.64 \\
\hline Estrés & & \\
\hline
\end{tabular}




\begin{tabular}{lll} 
Fomento de la confianza & 1 & 1.64 \\
\hline Autoestima & 1 & 1.64 \\
\hline Creatividad & 1 & 1.64 \\
\hline Síndrome de burnout & 1 & 1.64 \\
\hline Resolución de problemas & 1 & 1.64 \\
\hline Toma de decisiones & 1 & 1.64 \\
\hline Satisfacción con el ocio & 1 & 1.64 \\
\hline Eficacia de entrenamiento & 1 & 1.64
\end{tabular}

Analizaremos cuales son los instrumentos de medida de inteligencia emocional encontrados en los artículos. Como podemos observar en la tabla 10, el instrumento más utilizado en los artículos es el Bar-On inventario de cociente emocional (EQ-i,) junto con el TEIQue, con un $10.90 \%$ seguido del, MSCEIT, Trait Meta-MoodScale (TMMS) y la escala de inteligencia emocional (EIS).

Tabla 10. No de trabajos según instrumento de medida

\begin{tabular}{|c|c|c|}
\hline Instrumentos & $\begin{array}{l}\text { No de } \\
\text { artículos }\end{array}$ & $\%$ \\
\hline $\begin{array}{l}\text { El Bar-On Inventario de Cociente Emocional } \\
(\text { EQ-i,) }\end{array}$ & 6 & 10.90 \\
\hline TEIQue & 6 & 10.90 \\
\hline Escala de Inteligencia Emocional (EIS) & 4 & 7.27 \\
\hline MSCEIT & 4 & 7.27 \\
\hline Trait Meta-Mood Scale (TMMS) & 4 & 7.27 \\
\hline El Eating Attitudes Test (EAT-26) & 2 & 3.64 \\
\hline $\begin{array}{l}\text { El Cuerpo del Ser Multidimensional Cuestio- } \\
\text { nario de Relaciones (MBSRQ) }\end{array}$ & 2 & 3.64 \\
\hline $\begin{array}{l}\text { El Inventario de Ansiedad Estado-Rasgo } \\
\text { (STAI) }\end{array}$ & 2 & 3.64 \\
\hline La Escala de emociones positivas y negativas & 2 & 3.64 \\
\hline Habilidades sociales (SSI) & 2 & 3.64 \\
\hline Schutte Emotional Intelligence Scale(SEIS) & 2 & 3.64 \\
\hline $\begin{array}{l}\text { Escala Multifactorial de la Inteligencia Emo- } \\
\text { cional (Meis) }\end{array}$ & 2 & 3.64 \\
\hline El inventario de riesgo y la emoción & 1 & 1.81 \\
\hline $\begin{array}{l}\text { Escala de Satisfacción Ocio y tiempo libre } \\
\text { (LSS) }\end{array}$ & 1 & 1.81 \\
\hline La participación libre de Participación (LPI), & 1 & 1.81 \\
\hline MCQ-Score & 1 & 1.81 \\
\hline Cuestionario Big Five(BFQ) & 1 & 1.81 \\
\hline $\begin{array}{l}\text { Características motivacionales (SMI y el } \\
\text { inventario de Costello) }\end{array}$ & 1 & 1.81 \\
\hline La Escala de Autoestima de Rosenberg (RSE) & 1 & 1.81 \\
\hline $\begin{array}{l}\text { Las actitudes y los estilos de liderazgo (LSS y } \\
\text { plomo) }\end{array}$ & 1 & 1.81 \\
\hline $\begin{array}{l}\text { Schema Questionnaire-Short Form, SQ-SF, } \\
\text { Young y Brown, 1994). }\end{array}$ & 1 & 1.81 \\
\hline Cuestionario síndrome de Maslach & 1 & 1.81 \\
\hline Los perfiles de fuerza mental & 1 & 1.81 \\
\hline
\end{tabular}

Prueba de estrategias de actuación (TOPS, y

Thomas, Murphy, \& Hardy, 1999)

$\begin{array}{lll}\text { Escala del estado de ánimo de Brunei } & 1 & 1.81\end{array}$

Regulación emocional y la Escala de Control $\quad 1 \quad 1-81$ (ERC)

Cuestionario de Competencia (ESCQ-45) $\quad 1 \quad 1.81$

Inventario de Competencia Emocional (ICE) $\quad 1 \quad 1.81$

Encuesta Clima Organizacional II (OCS II) $\quad 1 \quad 1.81$

Para finalizar nos parecía interesante comentar las modalidades deportivas en las cuales se ha trabajado. Siendo con los atletas con los que más se ha trabajado o se le ha instruido en Inteligencia emocional contando con un $34.48 \%$ del total de modalidades que se han ocupado en este estudio. Seguida muy de cerca de entrenadores (natación, atletismo y tenis) los cuales también eran instruidos en la importancia de que tanto para ellos como para los deportistas era necesario para su mejora del rendimiento la inteligencia emocional. Y en tercer lugar se encontraría el cricket.

Tabla 11. Modalidad deportiva

Modalidad Deportiva $\quad$ No de \%

$\begin{array}{ccc} & \text { artículos } & \\ \text { Atletas } & 10 & 34.48\end{array}$

Entrenadores(natación, atletismo y tenis) $\quad 5 \quad 17.24$

$\begin{array}{lll}\text { Cricket } & 3 & 10.34\end{array}$

$\begin{array}{lll}\text { Baloncesto } & 2 & 6.90\end{array}$

$\begin{array}{lll}\text { Judo } & 2 & 6.90\end{array}$

$\begin{array}{lll}\text { Balonmano } & 1 & 3.57\end{array}$

$\begin{array}{lll}\text { Golf } & 1 & 3.57\end{array}$

$\begin{array}{lll}\text { Montañeros } & 1 & 3.57\end{array}$

$\begin{array}{lll}\text { Taekwondo } & 1 & 3.57\end{array}$

$\begin{array}{lll}\text { Tenis de mesa } & 1 & 3.57\end{array}$

$\begin{array}{lll}\text { Hockey } & 1 & 3.57\end{array}$

$\begin{array}{lll}\text { Béisbol } & 1 & 3.57\end{array}$

\section{Discusión y Conclusiones}

El fin de la presente investigación se ha centrado en examinar las publicaciones científicas sobre inteligencia emocional y deporte. Esto se ha realizado a través de un análisis bibliométrico y utilizando para ello las base de datos PsycINFO, SPORTDiscus, Academic Search Premier, Web of Science y Medline.

Como comentábamos al inicio de este trabajo, los resultados que ofrecemos son la primera fase de un estudio más amplio que deseamos realizar arrancando a partir de aquí una nueva línea de investigación que hasta el momento no habíamos trabajado. Una vez conseguidos los resultados del análisis bibliométrico, podemos detectar algunos datos bási- 
cos que deben ser atendidos en las propuestas investigadoras futuras que versen sobre la inteligencia emocional en el contexto deportivo. Para ello deberemos atender a las siguientes conclusiones que hemos destacado del presente estudio.

El estudio sobre Inteligencia Emocional y el Deporte ha sido escaso. Lo cual es llamativo si tenemos en cuenta la importancia que este proceso tiene en otros ámbitos como el educativo y a nivel laboral y no vemos que no es tan estudiado a nivel de la actividad física y deporte. Por un lado, creemos de vital importancia que el deportista que posee una buena inteligencia emocional podrá conseguir o mejorar su rendimiento con respecto aquel deportista que no lo posea. Esta aparición de la inteligencia emocional en los deportistas puede provocar menos bajas deportivas, aumento del rendimiento deportivo y menos abandonos, lo que hace que sea motivo de interés de estudio tanto para el mundo deportivo como para la sociedad en general. Por otro lado, la inteligencia emocional es un constructo de la personalidad que actúa como factor determinante cuando el deportista se encuentra en situaciones de presión y debe tomar decisiones en decimas de segundo lo que genera grandes picos de estrés, por lo que consideramos necesario para cualquier deportista y sobre todo para los deportistas de élite el estar entrenados a este nivel. Además, la mayor parte de estas investigaciones son de naturaleza empírica, y se echa en falta la presencia de trabajos que aporten una mayor integración teórica sobre el campo y que expliciten cuáles son los modelos formales que los sustentan.

Es a partir de 1995 cuando aparece con la obra Inteligencia Emocional de Goleman cuando se inicia y amplia el estudio de la inteligencia emocional en medios tanto científicos como no científicos produciéndose un aumento del interés social y científico sobre los procesos emocionales pero no aparecerá en el contexto deportivo hasta el 2001, donde empiezan aparecer los primeros trabajos. Pero sobre todo, a partir del 2009, es cuando se inicia estos estudios de manera más importante. En esta última década, se ha experimentado un aumento progresivo de la producción científica de este constructo. Aunque hay que reconocer este aumento, el número de trabajos es escaso para esta variable. A pesar de esto, parece existir un interés cada vez mayor en esta temática aunque hay que reconocer que es un ámbito virgen y con posibilidades de futura producción.

La producción total para inteligencia emocional y deporte fue de 60 publicaciones, lo que supone una media de 1.85 autores por trabajo respectivamente. Una producción baja, ya que el número de autores ocasionales o transeúntes podemos observar que fue alto. Hay que tener presente que el número de estos autores (con una sola publicación en el estudio) es un índice que da idea de la consolidación de la actividad científica en un país, área o disciplina (Shubert y Glänzel, 1991). Por consiguiente, un porcentaje alto de autores ocasionales sería preocupante y su disminución sería lo deseable. Esto es lo que sucede con nuestro constructo. Por tanto, que sean tan escasos en este campo los grandes productores y/o productores moderados, viene a indicar que aún no está suficientemente establecida el área de investigación y que frecuentemente son personas que tienen una vinculación esporádica con un grupo de investigación y que por tanto firman conjuntamente con otros.

Opinamos que es algo bajo el número de autores que publican más de una vez en esta temática, quizás justificado por la poca atención que la investigación sobre esta variable en deporte ha tenido en la comunidad científica.

La producción de los autores sigue la siguiente tendencia: se recoge una serie reducida de autores que son los que más publican y, después, una gran cantidad de autores que publican un trabajo o dos. Esto nos indica que los autores que más publican son los que tiene más posibilidades de volver a publicar un nuevo trabajo. Aparece A.M. Lane, y T.J. Devonport, con 8 y 6 artículos respectivamente siendo los autores más productivos, convirtiéndose en referencias obligadas de cualquier línea de investigación sobre inteligencia emocional y deporte que se diseñe. Nos encontramos además con una tendencia significativa a la colaboración como lo prueba que la mayoría de trabajos publicados hayan sido realizados por colaboración entre 2 o 3 autores. Esto nos indica que en esta variable se trabaja normalmente en colaboración, formando equipos de varias personas.

Además, desde una perspectiva geográfica, la mayoría de los trabajos son realizados en EEUU y en el Reino Unido. En el resto de países, la producción es mucho menor o por lo menos se publican mucho menos trabajos.

Destacamos que la mayor productividad por Universidades se encuentra en la University of Wolverhampton que corresponde al Reino Unido lo cual no es de extrañar ya que como hemos comentado antes se acerca bastante a la producción de EEUU.

El tipo de documento más utilizado es el artículo de revista. Las tesis con muy poca representatividad, nos da una idea del poco desarrollo de estas variables en el deporte a nivel universitario, por lo que entendemos que es un campo donde se pueden realizar aportaciones interesantes tanto desde la perspectiva teórica como práctica. De estos artículos destaca por mayoría la investigación empírica sobre la teórica. Es decir, predominan las investigaciones que acumulan datos $y$, comparativamente, escasean los trabajos dedicados a la revisión con finalidad integradora.

El International Journal of Sports Science \& Coaching es la revista donde aparecen el mayor número de artículos correspondiéndole el $18.1 \%$ de la producción total de artículos.

Nos parece interesante resaltar algunas de las variables que se han trabajado en los artículos publicados haciéndonos una idea de la línea que se está siguiendo en este momento y 
por donde los autores productores están directamente en el contexto deportivo. Concretamente las variables con las que más se ha estudiado son el liderazgo, el rendimiento deportivo seguido de tres variables que coinciden en su aparición que son el desarrollo y la mejora de su inteligencia emocional, las estrategias de autorregulación y la ansiedad.
Con respecto a los instrumentos de evaluación los más utilizados, son El Bar-On Inventario de Cociente Emocional (EQ-i,), el TEIQue, la Escala de Inteligencia Emocional (EIS), el MSCEIT y el Trait Meta-Mood Scale (TMMS) aunque como observamos aparecen multitud de instrumentos en los diferentes estudios.

\section{Referencias bibliográficas}

1. Biddle, S.J. y Hill, A.B.(1992). Relationship between attributions and emotions in a laboratory based sporting contest. Journal of Sport Sciences, 1(10), 65-75.

2. Dosil, J. (2004).Psicología de la Actividad Física y del Deporte. Madrid: McGraw-Hill.

3. Goleman,D. (1995). Emotional Intelligence. New York: Bantam Books.

4. Gluch,P.(1993).The use of music in preparing for sport performance. Contemporary Thought on Performance Enhancement, 2 (2); 33-53.

5. Hanin,Y.L.(1993). Optimal performance in tops athletes. En S. Serpa, et al.,(eds), VIII World Congress of Sport Psychology. Lisboa, Portugal: Internacionl Society.

6. Hanin,Y.L.(1995).Assessment of performance emotions in sport. En J. Vitasolo, y U. Kujala, (eds), The way to win: Internacional Congress on Applied Research in Sport.9-11 Aug.1994. Helsinik, Finlandia: Finnish Society for Research in Sport and Physical Education.

7. Hanin,Y.L y Sija, P.(1995). Performance affect in junior ice hockey players: An application of the individual zones of optimal functioning model. Sport Psychologist, 2(9);169-187.

8. Liukkonen, J.(1995). Regulation of performance emotions in sport. En J. Vitasoloy U. Kujala,(eds), The way to win: Internacional Congress on Applied Research in Sport. 9-11 Aug.1994. Helsinik, Finlandia:Finnish Society for Research in Sport and Physical Education.

9. Lorenzo, J. (1997) Psicología del deporte. Madrid: Biblioteca Nueva

10. Marciano, G. (1989). Movimiento de emozioni. Scuoladello
Sport, 17(8);62-65.

11. Markowska,L.; Nowicki, D.; Stupnick,R. y Lesinska, A. (1991). The effects of relaxation training on psychohormonal reactions to examination stress. Biological of Sport, 2(8); 77-86.

12. Ranglin,J.S.(1995). Performance emotions in sport; Concepts and models. En Vitasolo, J.yKujala,U.(eds), The way to win: Internacional Congress on Applied Research in Sport.9-11 Aug.1994. Helsinik, Finlandia: Finnish Society for Research in Sport and Physical Education.

13. Riera, J. (1985). Introducción a la psicología del deporte. Barcelona: Martínez Roca.

14. Silva, J. (1984). Personality and sport performance: Controversy and challenge. En Silva J. y R. Weinberg (eds.), Psychological foundations of sport. Champaign, Illinois: Human Kinetics.

15. Smith, R. y Smoll, F. (1991) Sports as natural laboratory for psychological research and intervention. En K. Vraiget al. (eds.), Psychology, sport and healthpromotion. Champaign, Illinois: Human Kinetics.

16. Spielberger, C.D. y Guerrero, R. (1975).IDARE: Inventario de ansiedad rasgo-estado.Manual instructivo de inventario de ansiedad rasgoestado. Mexico: El Manual Moderno.

17. Shubert, A. y Glänzel, W. (1991). Publication dynamics: Models and indicators. Scientometrics, 20(1), 317-331.

18. Thomas,P.R.(1994).Psychological and psychomotor skills associated with performance in golf. Sport Psychologist, 1(8):73-86. 